\title{
EFFECTS OF MILD TRYPSIN TREATMENT ON THE PASSIVE TENSION GENERATION AND CONNECTIN SPLITTING IN STRETCHED SKINNED FIBERS FROM FROG SKELETAL MUSCLE
}

\author{
Toshitada Yoshioka ${ }^{1}$, Hideo Higuchi ${ }^{2}$, Sumiko Kimura ${ }^{3}$, Kazuyo Ohashi ${ }^{3}$, \\ Yoshiki UMAZUME ${ }^{2}$ and KosCaK MARUYAMA ${ }^{3}$ \\ ${ }^{1}$ Department of Physiology, Tokai University School of Medicine, Kanagawa 259-11, ${ }^{2}$ Department of \\ Physiology, The Jikei University School of Medicine, Tokyo 105, and ${ }^{3}$ Department of Biology, Faculty of \\ Science, Chiba University, Chiba 260, Japan
}

\begin{abstract}
When skinned fibers from frog semitendinous muscle were mildly treated with trypsin, passive tension generation was remarkably reduced, and the hydrolysis of $\alpha$-connectin to $\beta$-connectin simultaneously occurred. On immunoelectron microscopy, splitting of the connectin filaments at the A-I junction area in a sarcomere was seen. These observations strongly suggest that connectin filaments are responsible for passive tension generation as a parallel elastic component in a sarcomere.
\end{abstract}

Since the pioneering work of skinned muscle fibers by Natori (10), it has been well established that an intramyofibrillar elastic structure is responsible for the resting tension generation by stretch beyond the overlap regions of thick and thin filaments in a sarcomere $(4,5,11)$. Recently, Higuchi and Umazume (2) have suggested a possible role of connectin, an elastic filamentous protein, in the elasticity by showing a drop in tension generation in muscle fibers whose myosin was partially dissolved. Connectin filaments connect myosin (thick) filaments to $\mathrm{Z}$ discs in myofibrils $(8,9)$. Connectin is very sensitive to proteolytic enzymes such as trypsin and chymotrypsin $(3,6)$. Connectin exists as $\alpha$ connectin $(2,800 \mathrm{kDa})$ in striated muscle, and its proteolytic product represents $\beta$-connectin $(2,100 \mathrm{kDa})(7)$. Tension generation in stretched skinned fibers easily drops also upon a mild treatment of fibers with trypsin ( $c f$. reference 11). In the present study, changes in passive tension generation, splitting of connectin, and changes in fine structures of myofibrils as seen by immunoelectron micros- copy, were simultaneously investigated during a mild treatment of skinned fibers with trypsin. The results show that hydrolysis of $\alpha$ connectin to $\beta$-connectin is closely related to a drop in passive tension generation in skinned fibers.

\section{MATERIALS AND METHODS}

\section{Preparation of Skinned Fibers}

Single muscle fibers dissected from semitendinous muscle of the frog (Rana castesbeiana) were mechanically skinned in a relaxing solution consisting of $90 \mathrm{mM} \mathrm{KCl}, 5.2 \mathrm{mM} \mathrm{MgCl}_{2}$, $4.3 \mathrm{mM} \mathrm{Na} \mathrm{F}_{2} \mathrm{ATP}, 4.0 \mathrm{mM}$ EGTA, and $10 \mathrm{mM}$ PIPES, $\mathrm{pH}$ 7.0. The resting tension of skinned fibers was measured as described (2).

About 10 and $40 \mathrm{~min}$ after stretching the skinned fibers to the sarcomere length of 3,4 and $5 \mu \mathrm{m}$, respectively, the fibers were incubated in the relaxing solution containing $0.25 \mu \mathrm{g} / \mathrm{ml}$ trypsin (Sigma, type III, 9,200 BAEE units/mg trypsin). After the skinned fibers were briefly digested by trypsin, they were fixed for $10 \mathrm{~min}$ with the relaxing solu- 


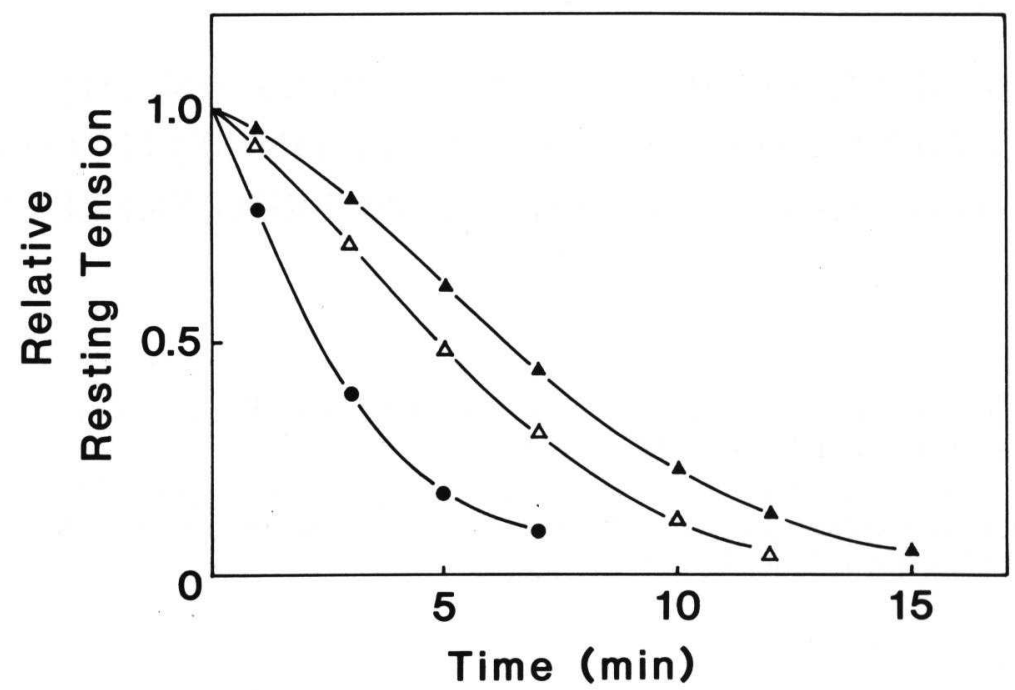

Fig. 1 Effect of trypsin treatment on the resting tension generation in skinned fibers from frog skeletal muscle. The treatment was performed in a relaxing solution containing trypsin $(0.25 \mu \mathrm{g} / \mathrm{ml})$ at $\mathrm{pH} 7.0$ and at $25^{\circ} \mathrm{C}$. $\bullet$, sarcomere length, $3 \mu \mathrm{m} ; \Delta, 4 \mu \mathrm{m} ; \Delta, 5 \mu \mathrm{m}$

tion containing $10 \%$ formalin. All the procedures were performed at $20^{\circ} \mathrm{C}$.

\section{Sodium Dodecyl Sulfate (SDS)-Gel Electrophoresis}

Two to three skinned fibers of $10-15 \mathrm{~mm}$ in length were gently suspended in about $0.2 \mathrm{ml}$ of the solution containing $10 \%$ SDS, $40 \mathrm{mM}$ dithiothreitol, $10 \mathrm{mM}$ EDTA, and $100 \mathrm{mM}$ Tris- $\mathrm{HCl}$ buffer, $\mathrm{pH}$ 8.0. After boiling for 2 min, the sample was briefly centrifuged, and the supernatant was subjected to gel electrophoresis using the $2 \%, 3 \%$ or $10 \%$ acrylamide gel essentially according to Weber and Osborn (12). The glass tube used was $2.5 \mathrm{~mm}$ in diameter and $6 \mathrm{~cm}$ in length.

\section{Immunoelectron Microscopy}

This was carried out as reported previously (9). The control and trypsin-treated fibers were fixed with formalin followed by the treatment with antiserum raised against native chicken breast muscle connectin. The thin sections were examined under a JEM 100C electron microscope.

\section{RESULTS}

Effect of Trypsin Treatment on Passive Tension Generation in Skinned Fibers

Mild trypsin treatment resulted in a rapid drop in passive tension generation in skinned fibers from frog skeletal muscle. In skinned fibers kept at a constant sarcomere length of $3 \mu \mathrm{m}$, and treated with trypsin $(0.25 \mu \mathrm{g} / \mathrm{ml})$ for $5 \mathrm{~min}$ at $20^{\circ} \mathrm{C}$, passive tension generation decreased to only $15 \%$ of the value in intact fibers (Fig. 1). In stretched fibers, $5 \mu \mathrm{m}$ in the sarcomere length, changes were less drastic; about 12 min were required before the tension was reduced to $15 \%$ of the initial value. The extent of reduction in tension was intermediate when the sarcomere length was $4 \mu \mathrm{m}$. The difference in trypsin sensitivity in fibers with different sarcomere lengths remains to be explained.

\section{SDS-Gel Electrophoresis of Trypsin-Treated Skinned Fibers}

Trypsin treatment of skinned fibers resulted in the gradual splitting of $\alpha$-connectin to $\beta$-connectin (Fig. 2A). In the intact skinned fibers, $\alpha$-connectin was more predominantly seen than $\beta$-connectin. These two connectin bands were identified as connectin by cross-reaction with an antiserum against chicken breast 
A

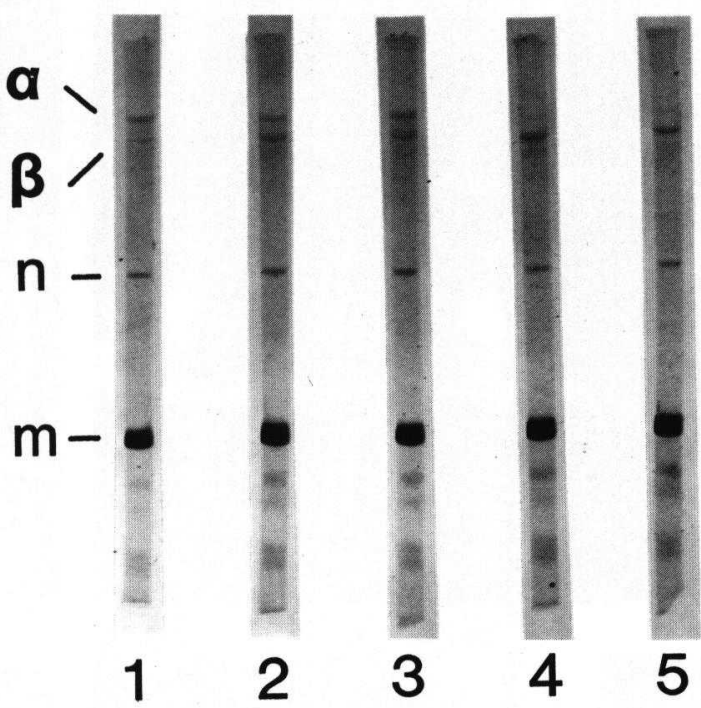

B

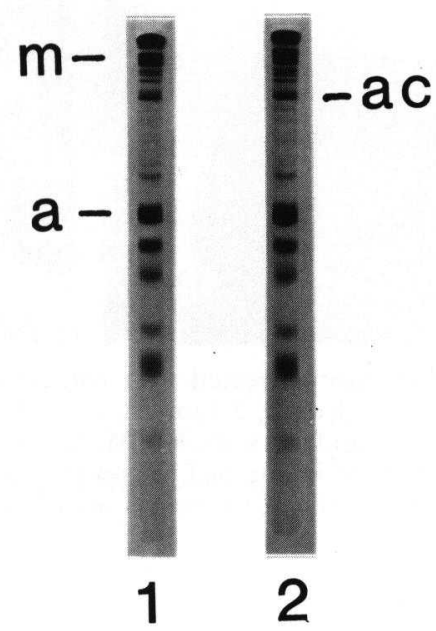

Fig. 2 SDS-gel electrophoresis patterns of trypsin-treated skinned fibers from frog skeletal muscle. Treated with trypsin as in Fig. 1. Sarcomere length, $3 \mu \mathrm{m}$. A: $2 \%$ Polyacrylamide gels. 1, intact; 2, treated with trypsin for $1 \mathrm{~min} ; 3$, for $3 \mathrm{~min} ; 4$, for $5 \mathrm{~min}$; and 5 , for $7 \mathrm{~min}$. B: $10 \%$ Polyacrylamide gels. 1 , intact; 2 , treated with trypsin for $7 \mathrm{~min}$. $\alpha, \alpha$-connectin; $\beta, \beta$ connectin; n, nebulin; m, myosin heavy chain; ac, $\alpha$-actinin; a, actin 

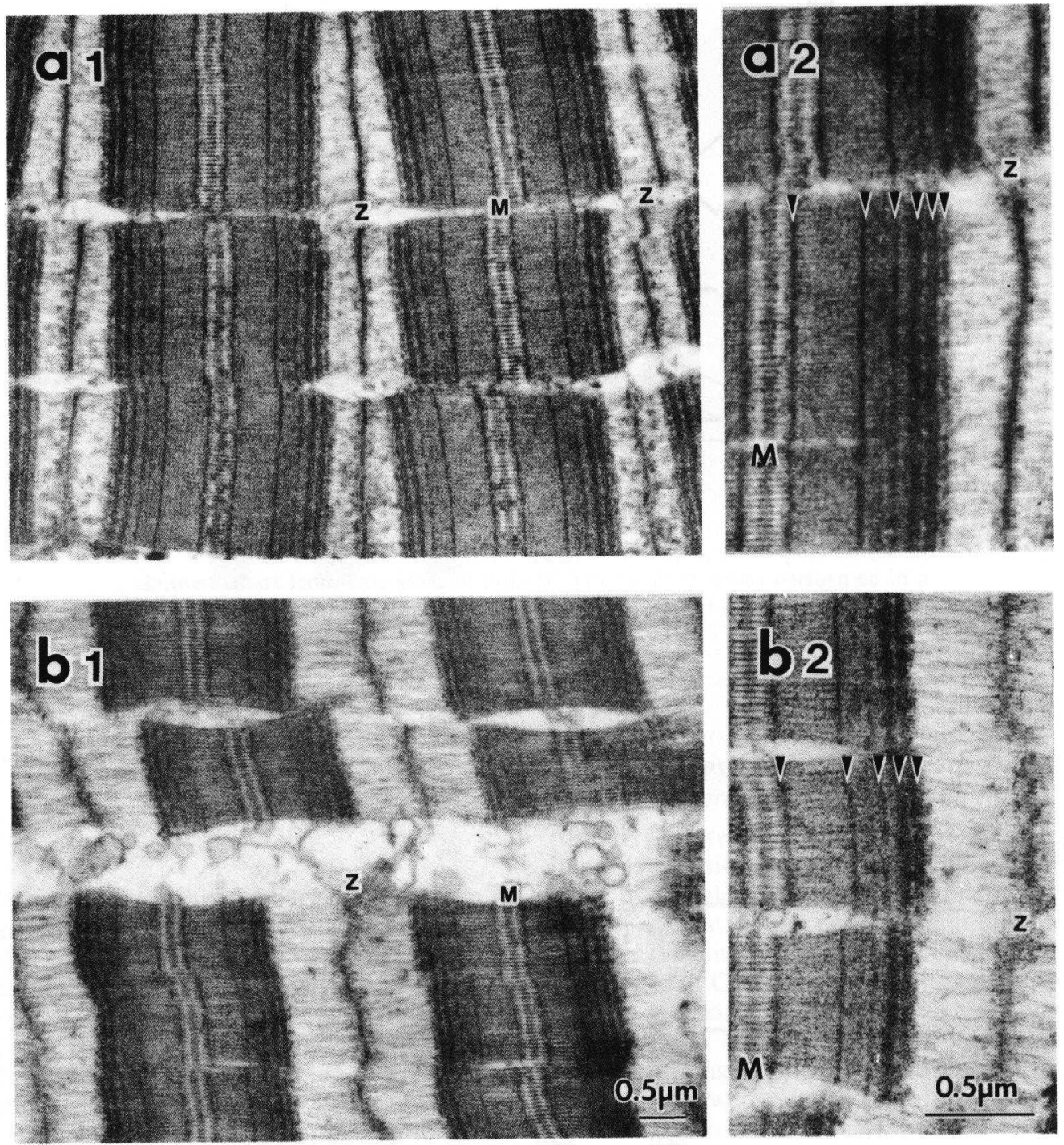

Fig. 3 Immunoelectron micrographs of myofibrils treated with anti-connectin antiserum. Skinned fibers were pretreated with trypsin. Sarcomere length, $2.5 \mu \mathrm{m}$. a, control; b, treated with trypsin for 5 min. Magnifications: $\mathrm{a} 1$ and b1 $\times 12,000 ; \mathrm{a} 2$ and b2, $\times 28,000$. M, M-line; Z, Z disc. In a1 and a2, six stripes with anti-connectin deposits can be seen in the half sarcomere, whereas only five stripes are visible in b1 and b2. The outer most stripe at the A-I junction area has been removed.

muscle connectin (9). Within $3 \mathrm{~min}$ at $20^{\circ} \mathrm{C}$ in the presence of trypsin $(0.25 \mu \mathrm{g} / \mathrm{ml}), \alpha$-connectin was largely hydrolyzed to $\beta$-connectin, and within $5 \mathrm{~min}$, further digestion to $1,900 \mathrm{kDa}$ fragment was observed. It should be noted that nebulin $(750 \mathrm{kDa})$, another trypsin-sensitive protein (6), did not show any sign of hydrolysis under the same conditions. Myosin also remained intact at least for $7 \mathrm{~min}$ under the present experimental conditions. No changes were observed in the protein banding pattern in $10 \%$ acrylamide gel after the mild trypsin treatment (Fig. 2B), suggesting that only connectin was split during the present trypsin treatment. The above results were obtained with fibers maintained at a sarcomere length of $3 \mu \mathrm{m}$. In fibers with sarcomere length of $5 \mu \mathrm{m}$, splitting of connectin 

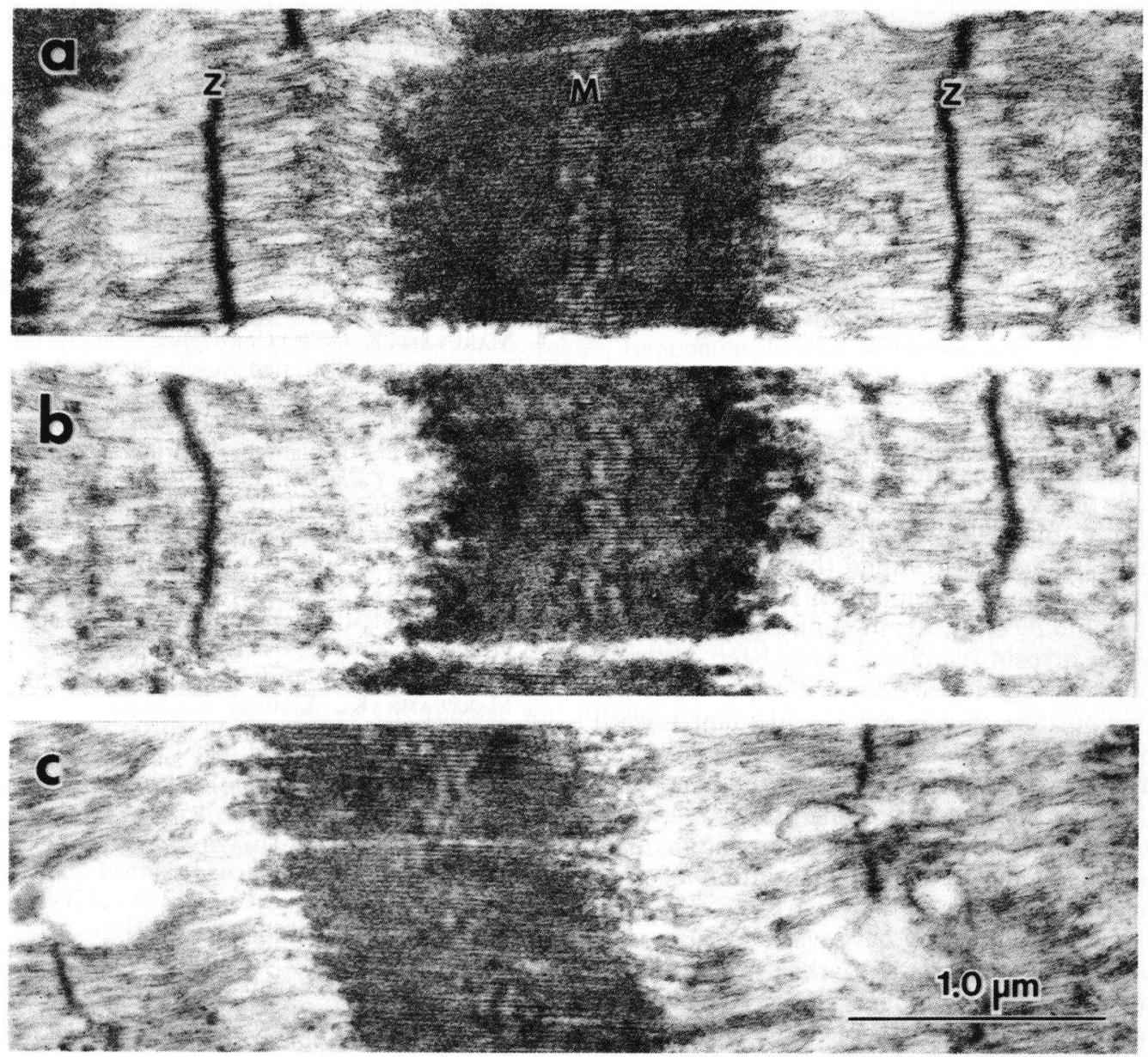

Fig. 4 Immunoelectron micrographs of myofibrils from skinned fibers pretreated with trypsin, and then treated with anti-connectin antiserum. Sarcomere length, $4 \mu \mathrm{m}$. a, control; b, treated with trypsin for $5 \mathrm{~min}$; and c, for 10 min. Magnifications: a, b, and c, $\times 30,000$. M, M-line; Z, Z disc

was somewhat delayed.

\section{Changes in Fine Structure of Anti-Connectin Antiserum-Treated Skinned Fibers}

Myofibrils of frog skeletal muscle treated with anti-connectin antiserum showed characteristic stripe patterns as previously reported (9). It has been postulated that connectin filaments link myosin filaments directly to $Z$ discs. It is of interest to examine the changes in the stripe patterns after the mild treatment with trypsin.

Fig. 3 shows immunoelectron micrographs of trypsin-treated myofibrils whose sarcomere length had been kept at $2.5 \mu \mathrm{m}$. It appeared that the stripe patterns did not change with the trypsin treatment. On closer examinations, however, the outermost stripe at the A-I junction area was found to be deteriorated, and frequently removed (the most righthand arrowhead in Fig. 3, a2 and b2). It thus appears that connectin filaments were cut off by tryptic digestion around the A-I junction area.

The stretched myofibrils (approximately $4 \mu \mathrm{m}$ in sarcomere length) did not show any regular stripe patterns due to dislocations of antigenic sites on connectin filaments (Fig. 4). Any particular changes related to connectin could not be seen after the trypsin treatment; the whole structure was more or less disor- 
dered ( $c f$. reference 11 ).

It should be noted that the trypsin treatment resulted in a decrease in density in the $Z$ discs (Figs. 2b and 3c). This may be attributed to a release of $\alpha$-actinin from the $Z$ discs during trypsin digestion of myofibrils (1). However, SDS-gel electrophoresis (Fig. 2B) showed that the band corresponding to $\alpha$ actinin ( $c f$. reference 9) did not change appreciably by the trypsin treatment under the present experimental conditions.

\section{DISCUSSION}

Resting tension generation of a myofibril is explained by assuming that elastic filaments link one $\mathrm{Z}$ disc to a neighboring disc. Connectin filaments are regarded to attach to myosin filament running through $\mathrm{Z}$ discs (8, 9 ). Based on the symmetrical stripe patterns of the binding sites of anti-connectin antibodies along myosin filaments, it has been assumed that one connectin filament starts from the mid-portion of one myosin filament toward one $\mathrm{Z}$ disc and the other toward another $\mathrm{Z}$ disc in one sarcomere. Higuchi and Umazume (2) have demonstrated that resting tension generation is decreased by removing myosin, which results in partial dissolution of connectin filaments.

The present study has revealed that passive tension generation was markedly reduced by a mild trypsin treatment through hydrolysis of $\alpha$-connectin $(2,800 \mathrm{kDa})$ to $\beta$-connectin $(2,100 \mathrm{kDa})$. On immunoelectron microscopy, possible splitting of connectin filaments at the A-I junction region was suggested, although further evidence is needed to specify the site of hydrolysis of $\alpha$-connectin.

Received for publication 2 April 1986; and in revised form 14 April 1986

\section{REFERENCES}

1. Goll D. E., Mommaerts W. F. H. M., Reedy M. K. and Seraydarian K. (1969) Studies on $\alpha$ - actinin-like proteins liberated during trypsin digestion of $\alpha$-actinin and of myofibrils. Biochim. Biophys. Acta 175, 174-194

2. Higuchi H. and Umazume Y. (1985) Localization of the parallel elastic components in frog skinned muscle fibers studied by the dissociation of the A- and I-bands. Biophys. J. 48, 137147

3. Kimura S., Yoshidomi H. and Maruyama K. (1984) Proteolytic fragments of connectin cause aggregation of myosin filaments but not of actin filaments. J. Biochem. 96, 1947-1950

4. Maruyama K. (1986) Connectin, an elastic filaments protein of striated muscle. Int. Rev. Cytol. 104, 81-114

5. Maruyama K. and Kimura S. (1985) Connectin, an elastic filament of vertebrate skeletal muscle. In Cell Motility: Regulation and Function (ed. Ishikawa H., Hatano S. and Sato H.) Univ. Tokyo Press, Tokyo, pp. 561-569

6. Maruyama K., Kimura M., Kimura S., Ohashi K., Suzuki K. and Katunuma N. (1981) Connectin, an elastic protein of muscle. Effects of proteolytic enzymes in situ. J. Biochem. 89, 711-715

7. Maruyama K., Kimura S., Yoshidomi H., Sawada H. and KiKuchi M. (1984) Molecular size and shape of $\beta$-connectin, an elastic protein of striated muscle. J. Biochem. 95, 1423-1433

8. Maruyama K., Sawada H., Kimura S., Ohashi K., Higuchi H. and Umazume Y. (1984) Connectin filaments in stretched skinned fibers of frog skeletal muscle. J. Cell Biol. 99, 1391-1397

9. Maruyama K., Yoshioka T., Higuchi H., Ohashi K., Kimura S. and Natori R. (1985) Connectin filaments link thick filaments and Z lines in frog skeletal muscle as revealed by immunoelectron microscopy. J. Cell Biol. 101, 2167-2172

10. NATORI R. (1954) The rôle of myofibrils, sarcoplasma and sarcolemma in muscle contraction. Jikeikai Med. J. 1, 18-28

11. Natori Rb., Umazume Y., Natori R. and YoshioKA T. (1980) The elastic structure of sarcomere. The relation of connectin filaments with thick and thin filaments. Jikeikai Med.J. 27, 83-97

12. WEBer K. and OsBorn M. (1969) The reliability of molecular weight determinations by dodecyl sulfate-polyacrylamide gel electrophoresis. $J$. Biol. Chem. 244, 4406-4412 